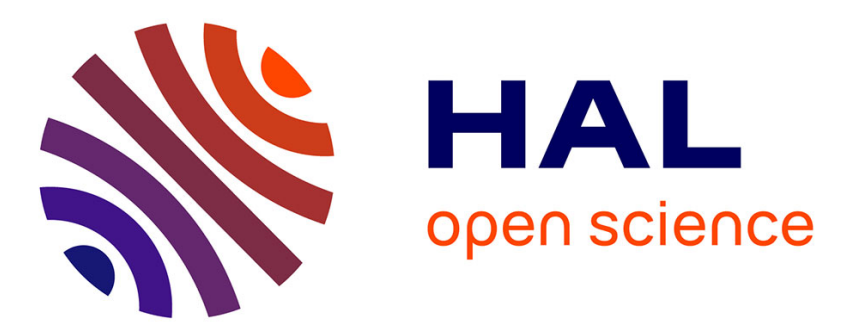

\title{
Existence of a directional Stokes drift in asymmetrical three-dimensional travelling gravity waves
}

\author{
Gérard Iooss, Pavel Plotnikov
}

\section{To cite this version:}

Gérard Iooss, Pavel Plotnikov. Existence of a directional Stokes drift in asymmetrical threedimensional travelling gravity waves. Comptes Rendus Mécanique, 2009, 10.1016/j.crme.2009.09.001 . hal-01265171

\section{HAL Id: hal-01265171 \\ https://hal.univ-cotedazur.fr/hal-01265171}

Submitted on 31 Jan 2016

HAL is a multi-disciplinary open access archive for the deposit and dissemination of scientific research documents, whether they are published or not. The documents may come from teaching and research institutions in France or abroad, or from public or private research centers.
L'archive ouverte pluridisciplinaire $\mathbf{H A L}$, est destinée au dépôt et à la diffusion de documents scientifiques de niveau recherche, publiés ou non, émanant des établissements d'enseignement et de recherche français ou étrangers, des laboratoires publics ou privés. 


\title{
Existence of a directional Stokes drift in asymmetrical three-dimensional travelling gravity waves
}

\author{
Gérard Iooss $^{\dagger}$, Pavel Plotnikov ${ }^{\ddagger}$ \\ † IUF, Université de Nice, Labo J.A.Dieudonné, 06108 Nice Cedex 02, France \\ †Lavrentyev Institute of Hydrodynamics, Lavryentyev pr. 15, Novosibirsk 630090, Russia \\ gerard.iooss@unice.fr, plotnikov@hydro.nsc.ru
}

September 4, 2009

\begin{abstract}
We consider periodic travelling gravity waves at the surface of an infinitely deep perfect fluid. The pattern is non symmetric with respect to the propagation direction of the waves and we consider a general non resonant situation. Defining a couple of amplitudes $\varepsilon_{1}, \varepsilon_{2}$ along the basis of wave vectors which satisfy the dispersion relation, following [8], travelling waves exist with an asymptyotic expansion in powers of $\varepsilon_{1}, \varepsilon_{2}$, for nearly all pair of angles made by the basic wave vectors with the critical propagation direction, and for values of the couple $\left(\varepsilon_{1}^{2}, \varepsilon_{2}^{2}\right)$ in a subset of the plane, with asymptotic full measure at the origin. We prove the remarkable property that on the free surface, observed in the moving frame, the propagation direction of the waves differs from the asymptotic direction taken by fluid particles, by a small angle which is computed.

Existence d'une dérive directionnelle de Stokes dans les vagues de gravité tri-dimensionnelles asymétriques. On considère les vagues périodiques à la surface d'une couche de fluide parfait, de profondeur infinie, soumise à la seule gravité. Le réseau bidimensionnel des périodes est pris non symétrique par rapport à la direction de propagation et on suppose ne pas être dans un cas résonant. On définit le couple d'amplitudes $\varepsilon_{1}, \varepsilon_{2}$ le long des deux vecteurs d'onde de base qui vérifient l'équation de dispersion. D'après [8], les vagues asymétriques existent et possèdent un développement asymptotique en puissances de $\left(\varepsilon_{1}, \varepsilon_{2}\right)$, pour presque tous les angles faits par les vecteurs d'onde de base avec la direction critique de propagation, et pour des valeurs de $\left(\varepsilon_{1}^{2}, \varepsilon_{2}^{2}\right)$ dans un sous-ensemble du quadrant ayant une mesure asymptotiquement pleine à l'origine. Nous montrons la propriété remarquable dans le référentiel relatif, qu'à la surface libre, la direction de propagation des ondes diffère de la direction asymptotique prise par les trajectoires des particules de fluide, d'un petit angle qu'on calcule.
\end{abstract}


Key words: potential perfect fluid flow; nonlinear water waves; travelling gravity waves; bifurcation theory; asymmetric 3D waves

Mots-clés: écoulements potentiels de fluides parfaits; vagues non linéaires; ondes progressives de gravité; théorie des bifurcations; vagues tri-dimensionnelles asymétriques

\section{Introduction}

We consider small-amplitude three-dimensional doubly periodic travelling gravity waves on the free surface of a perfect fluid. These waves are steady in a frame moving with the velocity of the wave $(-\mathbf{c}$ in the absolute reference frame). The fluid layer is supposed to be infinitely deep, and the flow is irrotational only subjected to gravity. The bifurcation parameters are the horizontal phase velocity, and the direction of propagation of the travelling waves.

In 1847 Stokes [11] gave a nonlinear theory of two-dimensional travelling gravity waves, computing formally the flow up to the cubic order of the amplitude of the waves. He showed that, in the moving frame, the average velocity of particles in the horizontal direction is slightly different (at second order in the amplitude of the waves) from the propagation velocity of the waves. In this note we show an analogous result for the common asymptotic direction taken by the trajectories of fluid particles in the moving frame (which we define as a "directional Stokes drift"), in the cases when the periodic pattern is asymmetric with respect to the propagation direction of the travelling waves. Formal calculations of solutions in power series of the amplitudes $\left(\varepsilon_{1}, \varepsilon_{2}\right)$ have a long history in literature (since the 50's for 3D travelling waves), see [3] for a recent review on water waves. However quite surprisingly, it seems that the phenomenon of directional Stokes drift is not mentioned yet, even for gravity-capillary travelling waves.

One serious theoretical difficulty here, is that we assume the absence of surface tension. Indeed the surface tension plays a major role in all existing proofs for three-dimensional travelling gravity-capillary waves (see [10], [1], [2], $[5],[4])$, and when the surface tension is very small, which is the case in many usual situations, this implies a reduced domain of validity of results strongly dependent on the existence of a non small surface tension. In our previous work [7] on three-dimensional travelling gravity waves, there is indeed no surface tension, but we restricted the study to the existence of diamond waves: the periodic lattice is a diamond lattice, and there are equal amplitudes at the leading order associated with the two basic wave vectors $K_{1}, K_{2}$ symmetric with respect to the propagation direction of the waves. We proved the existence of bifurcating diamond gravity waves, symmetric with respect to the propagation direction of the waves. In such a case the horizontal projection of fluid particles trajectories is asymptotically in the direction of propagation velocity, which is the bissectrix of the angle made by $K_{1}$ and $K_{2}$.

In the present work, we consider asymmetrical travelling gravity waves, which implies that the basic wave vectors $K_{1}$ and $K_{2}$ have not the same length, and 
given the two amplitudes $\left(\varepsilon_{1}, \varepsilon_{2}\right)$ on the basic modes, the direction of propagation $\mathbf{u}$ of the waves is part of the unknown. As in the symmetrical case, there is a small divisor problem which leads to the use of the Nash-Moser implicit function theorem. The existence of asymmetrical gravity waves is proved in [8] (see Theorem 2.2 below) for nearly all choices of angles $\theta_{1}, \theta_{2}$ made by the non symmetric wave vectors $K_{1}, K_{2}$ with the direction $\mathbf{u}_{0}$ of the critical velocity, and for values of the square of the amplitudes $\left(\varepsilon_{1}^{2}, \varepsilon_{2}^{2}\right)$ on these wave vectors, in a set for which 0 is a Lebesgue point ( 0 corresponds to the critical value $\mathbf{c}_{c}$ of the velocity vector $\mathbf{c}$ ). Then Lemma 3.1 establishes the existence of a small angle between the propagation direction of the waves and the asymptotic direction of fluid particle trajectories in the frame moving with the velocity of the waves.

\section{Formulation of the problem}

Let us denote by $\varphi$ the potential defined by

$$
\varphi=\phi-\mathbf{c} \cdot X,
$$

where $\phi$ is the usual velocity potential in the moving frame (the rest state is then $\varphi=0), X=\left(x_{1}, x_{2}\right)$ is the 2 -dim horizontal coordinate, $x_{3}$ is the vertical coordinate, and the fluid region is

$$
\Omega=\left\{\left(X, x_{3}\right):-\infty<x_{3}<\eta(X)\right\},
$$

which is bounded by the free surface $\Sigma$ defined by

$$
\Sigma=\left\{\left(X, x_{3}\right): x_{3}=\eta(X)\right\} .
$$

We choose $|\mathbf{c}|$ for the velocity scale, and $L$ for a length scale (to be chosen below), and we still denote by $\left(X, x_{3}\right)$ the new coordinates, and by $\varphi, \eta$ the unknown functions. Now defining the parameter $\mu=\frac{g L}{c^{2}}$, where $g$ denotes the acceleration of gravity, and $\mathbf{u}$ the unit vector in the direction of $\mathbf{c}$, the system reads

$$
\begin{aligned}
\Delta \varphi & =0 \text { in } \Omega, \\
\nabla_{X} \eta \cdot\left(\mathbf{u}+\nabla_{X} \varphi\right)-\frac{\partial \varphi}{\partial x_{3}} & =0 \text { on } \Sigma, \\
\mathbf{u} \cdot \nabla_{X} \varphi+\frac{(\nabla \varphi)^{2}}{2}+\mu \eta & =0 \text { on } \Sigma, \\
\nabla \varphi & \rightarrow 0 \text { as } x_{3} \rightarrow-\infty .
\end{aligned}
$$

We specialize our study to spatially periodic 3-dimensional travelling waves, i.e. the solutions $\eta$ and $\varphi$ are periodic in $X$. There are two independent wave vectors $K_{1}, K_{2} \in \mathbb{R}^{2}$ generating a lattice

$$
\Gamma=\left\{\mathbf{k}=n_{1} K_{1}+n_{2} K_{2}: n_{j} \in \mathbb{Z}, j=1,2\right\},
$$


and we consider in the further analysis, a lattice $\Gamma$ generated by the wave vectors

$$
K_{1}=\left(1, \tau_{1}\right), K_{2}=\lambda\left(1,-\tau_{2}\right) .
$$

This means that we have chosen the length scale $L$ for having 1 in the first component of $K_{1}$, and $\tau_{1}, \tau_{2}$ measure the angles between the wave vectors $K_{1}, K_{2}$ and the $x_{1}$ axis. We can reduce the above system for $(\varphi, \eta)$ to a system of two scalar equations in choosing the new unknown function (following V.E.Zakharov)

$$
\psi(X)=\varphi(X, \eta(X))
$$

and we define the Dirichlet-Neumann operator $\mathcal{G}_{\eta}$ by

$$
\begin{aligned}
\mathcal{G}_{\eta} \psi & =\left.\sqrt{1+(\nabla \eta)^{2}} \frac{d \varphi}{d n}\right|_{x_{3}=\eta(X)} \\
& =\left.\frac{\partial \varphi}{\partial x_{3}}\right|_{x_{3}=\eta(X)}-\nabla \eta \cdot \nabla_{X} \varphi
\end{aligned}
$$

where $n$ is normal to $\Sigma$, exterior to $\Omega$, and $\varphi$ is the solution of the $\eta$ - dependent Dirichlet problem

$$
\begin{aligned}
\Delta \varphi & =0, \quad x_{3}<\eta(X) \\
\varphi & =\psi, \quad x_{3}=\eta(X), \\
\nabla \varphi & \rightarrow 0 \text { as } x_{3} \rightarrow-\infty .
\end{aligned}
$$

This definition of $\mathcal{G}_{\eta}$ follows [9] and insures the selfadjointness and positivity of this linear operator in $L^{2}\left(\mathbb{R}^{2} / \Gamma\right)$ (see [7]). Now the system to solve reads

$$
\mathcal{F}(U, \mu, \mathbf{u})=0, \quad \mathcal{F}=\left(\mathcal{F}_{1}, \mathcal{F}_{2}\right),
$$

where $U=(\psi, \eta)$, and

$$
\begin{aligned}
\mathcal{F}_{1}(U, \mathbf{u})= & : \mathcal{G}_{\eta}(\psi)-\mathbf{u} \cdot \nabla \eta \\
\mathcal{F}_{2}(U, \mu, \mathbf{u})= & : \mathbf{u} \cdot \nabla \psi+\mu \eta+\frac{(\nabla \psi)^{2}}{2}+ \\
& -\frac{1}{2\left(1+(\nabla \eta)^{2}\right)}\{\nabla \eta \cdot(\nabla \psi+\mathbf{u})\}^{2} .
\end{aligned}
$$

The mapping $\mathcal{F}(\cdot, \mu, \mathbf{u})$ is equivariant under translations of the plane:

$$
\mathcal{T}_{\mathbf{v}} \mathcal{F}(U, \mu, \mathbf{u})=\mathcal{F}\left(\mathcal{T}_{\mathbf{v}} U, \mu, \mathbf{u}\right)
$$

where

$$
\mathcal{T}_{\mathbf{v}} U(X)=U(X+\mathbf{v})
$$

and it is also equivariant with respect of the symmetry $\mathcal{S}_{0}$ defined by the following representation of the symmetry with respect to 0

$$
\left(\mathcal{S}_{0} U\right)(X)=(-\psi(-X), \eta(-X)) .
$$

The first result proved in [8], is the following 
Lemma 2.1 For any fixed integer $N \geq 3$, and $\boldsymbol{\tau}=\left(\tau_{1}, \tau_{2}\right) \in \mathbb{R}^{+2} \backslash\{0\}$, we define

$$
\mu_{c}=\left(1+\tau_{1}^{2}\right)^{-1 / 2}, \lambda=\left(\frac{1+\tau_{2}^{2}}{1+\tau_{1}^{2}}\right)^{1 / 2},
$$

and assume (non resonance condition) that the dispersion equation

$$
\mu_{c}|\mathbf{k}|-\left(\mathbf{k} \cdot \mathbf{u}_{0}\right)^{2}=0
$$

where $\mathbf{u}_{0}=(1,0)$, has only the solutions $\mathbf{k}= \pm K_{1}, \pm K_{2}, \mathbf{0}$, where $K_{1}=\left(1, \tau_{1}\right), K_{2}=$ $\lambda\left(1,-\tau_{2}\right)$, and $\mathbf{k} \in \Gamma$. Then, for any $\varepsilon=\left(\varepsilon_{1}, \varepsilon_{2}\right) \in \mathbb{R}^{+2}$, there is a family of approximate asymmetric travelling waves $\mathcal{T}_{\mathbf{v}} U_{N}(\varepsilon), \mathbf{v} \in \mathbb{R}^{2}$, where $U_{N}(\varepsilon)$ is given by the expansion

$$
\begin{aligned}
U_{N}(\varepsilon) & =\sum_{1 \leq p+q \leq N} \varepsilon_{1}^{p} \varepsilon_{2}^{q} U_{p q}, \\
\mu_{N}(\varepsilon) & =\mu_{c}+\sum_{1 \leq p+q \leq N / 2} \varepsilon_{1}^{2 p} \varepsilon_{2}^{2 q} \mu_{p q} \in \mathbb{R}^{+}, \\
\mathbf{u}_{N}(\boldsymbol{\varepsilon}) & =\mathbf{u}_{0}+\sum_{1 \leq p+q \leq N / 2} \varepsilon_{1}^{2 p} \varepsilon_{2}^{2 q} \mathbf{u}_{p q} \in \mathbb{S}_{1},
\end{aligned}
$$

with $X \mapsto U_{p q}(X)$ periodic and $\mathcal{C}^{\infty}$, such that $U_{p q}=\left(\psi_{p q}, \eta_{p q}\right)$ satisfies $\psi_{p q}(-X)=$ $-\psi_{p q}(X), \eta_{p q}(-X)=\eta_{p q}(X)$ and

$$
\begin{aligned}
& U_{10}=\left(\sin \left(K_{1} \cdot X\right), \frac{-1}{\mu_{c}} \cos \left(K_{1} \cdot X\right)\right), \\
& U_{01}=\left(\sin \left(K_{2} \cdot X\right), \frac{-\lambda}{\mu_{c}} \cos \left(K_{2} \cdot X\right)\right), \\
& \mu_{10}=\alpha_{1}, \mu_{01}=\alpha_{2}, \mathbf{u}_{10}=\left(0, \beta_{1}\right), \mathbf{u}_{01}=\left(0, \beta_{2}\right),
\end{aligned}
$$

the coefficients $\alpha_{j}$ and $\beta_{j}$ being explicit analytic functions of $\left(\tau_{1}, \tau_{2}\right)$ (explicitly given in [8]). Moreover

$$
\mathcal{F}\left(U_{N}(\varepsilon), \mu_{N}(\varepsilon), \mathbf{u}_{N}(\varepsilon)\right)=O\left(|\varepsilon|^{N+1}\right) .
$$

We notice that the non resonance condition is in fact a condition on $\left(\tau_{1}, \tau_{2}\right)$. It is indeed satisfied for a full measure set in $\mathbb{R}^{+2}$. Notice that if we make $\tau_{1}=\tau_{2}$, and $\varepsilon_{1}=\varepsilon_{2}$ we recover the result on diamond waves [7]. This Lemma may be proved by using a standard formal Lyapunov-Schmidt process, taking care of the 4-dimensional kernel of the linearized operator and in taking benefit of the symmetries $\mathcal{T}_{\mathbf{v}}$ and $\mathcal{S}_{0}$.

The following theorem is the main result of the paper [8]:

Theorem 2.2 Let us choose arbitrary integers $l \geq 34, N \geq 8$ and a real number $0<\delta<1$. Then, there is a full measure subset $\mathcal{T}$ of $\mathbb{R}^{+2}$ such that for any 
$\boldsymbol{\tau}=\left(\tau_{1}, \tau_{2}\right) \in \mathcal{T}$ there exists a subset $\mathcal{E}(\boldsymbol{\tau})$ of the quadrant $\left\{\left(\varepsilon_{1}^{2}, \varepsilon_{2}^{2}\right) \in \mathbb{R}^{+2}\right\}$ for which 0 is a Lebesgue point, i.e.

$$
\frac{2}{\epsilon^{2}} \operatorname{meas}\left(\mathcal{E}(\boldsymbol{\tau}) \cap\left\{\varepsilon_{1}^{2}+\varepsilon_{2}^{2}<\epsilon\right\}\right) \rightarrow 1 \text { as } \epsilon \rightarrow 0 .
$$

Moreover, for $\delta<\varepsilon_{1} / \varepsilon_{2}<1 / \delta$ and $\varepsilon=\left(\varepsilon_{1}, \varepsilon_{2}\right) \in \mathcal{E}(\boldsymbol{\tau})$, the system (2.2, 2.3,2.4) has a unique solution of the form

$$
\begin{aligned}
U & =U_{N}(\varepsilon)+|\varepsilon|^{N / 2} \breve{U}(\varepsilon) \in \mathbb{H}_{(S)}^{l}\left(\mathbb{R}^{2} / \Gamma\right), \\
\mu & =\mu_{N}(\varepsilon)+|\varepsilon|^{N / 2} \breve{\mu}(\varepsilon) \in \mathbb{R}^{+}, \\
\mathbf{u} & =\mathbf{u}_{N}(\varepsilon)+|\varepsilon|^{N / 2} \breve{\mathbf{u}}(\varepsilon) \in \mathbb{S}_{1},
\end{aligned}
$$

where $(\breve{U}(\varepsilon), \breve{\mu}(\varepsilon), \breve{\mathbf{u}}(\varepsilon)) \in \mathbb{H}_{(S)}^{l}\left(\mathbb{R}^{2} / \Gamma\right) \times \mathbb{R} \times \mathbb{R}^{2}$ is a Lipschitz function of $\varepsilon$, and where $\mathbb{H}_{(S)}^{l}\left(\mathbb{R}^{2} / \Gamma\right)$ means that $\psi$ and $\eta$ belong to a Sobolev class of periodic functions of order $l, \psi$ and $\eta$ being respectively odd and even in $X$.

The proof of this theorem is quite technical due to the occurence of a well known small divisor problem occuring in deriving the expansion given in Lemma 2.1, and follows the same structure as the proof of the main theorem in [6] and in [7]. As in [7], the above result expressed in terms of bifurcation analysis, provides a branch of solutions bifurcating from a non isolated eigenvalue 0 in the spectrum of the linearized operator at the origin. The additional difficulty here is due to the asymmetry of solutions, which precisely induces the phenomenon of Stokes directional drift explained below. The key point here is that, for proving the above theorem, we use a diffeomorphism of the torus which transforms the horizontal projection of the fluid particles trajectories (integral curves of a velocity field $V$ ), into straight lines. For being able to do this we need to assume that the rotation number $\rho$ of the horizontal velocity field $V$ satisfies a diophantine condition of the form

$$
\left|k_{1}+\rho k_{2}\right| \geq \frac{c}{|\mathbf{k}|^{1+\alpha}}
$$

where $0<\alpha<1 / 2$ is fixed, and for any $\mathbf{k}=\left(k_{1}, k_{2}\right) \in \mathbb{Z}^{2} \backslash\{\mathbf{0}\}$.

\section{Directional Stokes drift}

We now prove the following

Lemma 3.1 (Directional Stokes drift) In the frame moving with the velocity of the waves, the horizontal projection of the asymptotic direction taken by fluid particles makes an angle $\sim \mu_{c}^{-2}\left\{-\tau_{1} \varepsilon_{1}^{2}+\lambda^{4} \tau_{2} \varepsilon_{2}^{2}\right\}$ with the direction of propagation of the waves. There is a special value of the ratio $\varepsilon_{1}^{2} / \varepsilon_{2}^{2} \sim \lambda^{4} \tau_{2} / \tau_{1}$ for which both directions are identical. 
The idea is to identify the slope $p$ giving the asymptotic direction of the integral curves of the velocity field $V$, just knowing that this common asymptotic direction exists thanks to Theorem 2.2.

The velocity field $V$ is the horizontal projection of the velocity of fluid particles $\mathbf{u}+\nabla_{X} \varphi$, given by

$$
\begin{aligned}
V & =\mathbf{u}+\nabla \psi-\mathfrak{b} \nabla \eta \\
\mathfrak{b} & =\frac{1}{1+(\nabla \eta)^{2}}\{\nabla \eta \cdot(\mathbf{u}+\nabla \psi)\}
\end{aligned}
$$

where $\mathfrak{b}$ denotes $\left.\frac{\partial \varphi}{\partial x_{3}}\right|_{x_{3}=\eta(X)}$. From the expressions given in Lemma 2.1, we notice that

$$
\mathbf{u}=\left(1, \beta_{1} \varepsilon_{1}^{2}+\beta_{2} \varepsilon_{2}^{2}\right)+O\left(|\varepsilon|^{4}\right),
$$

and we find

$$
\begin{aligned}
V_{1}= & 1+\varepsilon_{1} \cos \left(X \cdot K_{1}\right)+\lambda \varepsilon_{2} \cos \left(X \cdot K_{2}\right)-\frac{\varepsilon_{1}^{2}}{2 \mu_{c}^{2}}-\frac{\varepsilon_{2}^{2} \lambda^{4}}{2 \mu_{c}^{2}}+O_{e}^{\prime}\left(|\varepsilon|^{2}\right), \\
V_{2}= & \tau_{1} \varepsilon_{1} \cos \left(X \cdot K_{1}\right)-\lambda \tau_{2} \varepsilon_{2} \cos \left(X \cdot K_{2}\right)+ \\
& +\left(\beta_{1}-\frac{\tau_{1}}{2 \mu_{c}^{2}}\right) \varepsilon_{1}^{2}+\left(\beta_{2}+\frac{\tau_{2} \lambda^{4}}{2 \mu_{c}^{2}}\right) \varepsilon_{2}^{2}+O_{e}^{\prime}\left(|\varepsilon|^{2}\right),
\end{aligned}
$$

where the terms $O_{e}^{\prime}\left(|\varepsilon|^{2}\right)$ are even in $X$, and represent a sum of terms of order $|\varepsilon|^{2}$ with 0 average, and higher order terms. Now we have for any integral line of the vector field $V$ in the plane $X=\left(x_{1}, x_{2}\right)$ :

$$
\begin{aligned}
\frac{d x_{2}}{d x_{1}}= & \frac{V_{2}}{V_{1}}=\tau_{1} \varepsilon_{1} \cos \left(X \cdot K_{1}\right)-\lambda \tau_{2} \varepsilon_{2} \cos \left(X \cdot K_{2}\right)+ \\
& +\left(\beta_{1}-\frac{\tau_{1}}{2 \mu_{c}^{2}}-\frac{\tau_{1}}{2}\right) \varepsilon_{1}^{2}+\left(\beta_{2}+\frac{\tau_{2} \lambda^{4}}{2 \mu_{c}^{2}}+\frac{\tau_{2} \lambda^{2}}{2}\right) \varepsilon_{2}^{2}+O_{e}^{\prime}\left(|\varepsilon|^{2}\right) .
\end{aligned}
$$

On the other hand, thanks to the existence of the diffeomorphism with a diophantine rotation number, we know that the integral lines take the form

$$
x_{2}=p x_{1}+q+\mathcal{Y}\left(X \cdot K_{1}, X \cdot K_{2}\right),
$$

where $\mathcal{Y}$ is $2 \pi$ - periodic in its arguments and is of order $O(|\varepsilon|)$ and $p$ is independent of the integral curve. Denoting by $x_{1}^{\prime}=X \cdot K_{1}, x_{2}^{\prime}=X \cdot K_{2}$, we have to identify the expression of $d x_{2} / d x_{1}$ given by (3.1) with the expression given by

$$
\left(1-\tau_{1} \partial_{x_{1}^{\prime}} \mathcal{Y}+\lambda \tau_{2} \partial_{x_{2}^{\prime}} \mathcal{Y}\right) \frac{d x_{2}}{d x_{1}}=p+\partial_{x_{1}^{\prime}} \mathcal{Y}+\lambda \partial_{x_{2}^{\prime}} \mathcal{Y}
$$

It results that

$$
\mathcal{Y}=\tau_{1} \varepsilon_{1} \sin \left(X \cdot K_{1}\right)-\tau_{2} \varepsilon_{2} \sin \left(X \cdot K_{2}\right)+O_{o}\left(|\varepsilon|^{2}\right)
$$


where the term $O_{o}\left(|\varepsilon|^{2}\right)$ is odd in $X$, and represents terms of order $|\varepsilon|^{2}$. Next order averaged gives

$$
\begin{aligned}
p & =\left(\beta_{1}-\frac{\tau_{1}}{2 \mu_{c}^{2}}-\frac{\tau_{1}}{2}\left(1+\tau_{1}^{2}\right)\right) \varepsilon_{1}^{2}+\left(\beta_{2}+\frac{\tau_{2} \lambda^{4}}{2 \mu_{c}^{2}}+\frac{\tau_{2} \lambda^{2}}{2}\left(1+\tau_{2}^{2}\right)\right) \varepsilon_{2}^{2}+O\left(|\varepsilon|^{4}\right) \\
& =\left(\beta_{1}-\frac{\tau_{1}}{\mu_{c}^{2}}\right) \varepsilon_{1}^{2}+\left(\beta_{2}+\frac{\tau_{2} \lambda^{4}}{\mu_{c}^{2}}\right) \varepsilon_{2}^{2}+O\left(|\varepsilon|^{4}\right) .
\end{aligned}
$$

Comparing this slope with the slope $\beta_{1} \varepsilon_{1}^{2}+\beta_{2} \varepsilon_{2}^{2}+O\left(|\varepsilon|^{4}\right)$ of the propagation direction $\mathbf{u}$, this leads to the result of the Lemma. It is a remarkable fact that there is a neutral value of the ratio between amplitudes $\varepsilon_{1} / \varepsilon_{2}$ for which both directions are identical.

\section{References}

[1] W.Craig, D.Nicholls. Traveling gravity water waves in two and three dimensions. EJMB/Fluids 21 (2002) 615-641.

[2] W.Craig, D.Nicholls. Travelling two and three-dimensional capillary gravity water waves. SIAM J. Math. Anal. 32 (2000) 323-359.

[3] F.Dias, C.Kharif. Nonlinear gravity and capillary-gravity waves. Annu. Rev. Fluid Mech. (1999), 31, 301-346.

[4] M.Groves, M.Haragus. A bifurcation theory for three-dimensional oblique travelling gravity-capillary water waves. J.Nonlinear Sci. 13 (2003), 397447.

[5] M.Groves, A.Mielke. A spatial dynamics approach to three-dimensional gravity-capillary steady water waves. Proc. Roy. Soc. Edin. A 131 (2001), 83-136.

[6] G.Iooss, P.Plotnikov, J.Toland. Standing waves on an infinitely deep perfect fluid under gravity. Arch. Rat. Mech. Anal. 177 (2005), 3, 367-478.

[7] G.Iooss, P.Plotnikov. Small divisor problem in the theory of threedimensional water gravity waves. Memoirs of AMS 200, 940 (2009).

[8] G.Iooss, P.Plotnikov. Asymmetrical three-dimensional travelling gravity waves. Preprint 2009

[9] D.Lannes. Well-posedness of the water-waves equations. J.Amer. Math. Soc. 18 (2005), 605-654.

[10] J.Reeder, M.Shinbrot. Three-dimensional, nonlinear wave interaction in water of constant depth. Nonlinear Anal., T.M.A., 5 (1981), 3, 303-323.

[11] G.G. Stokes. On the theory of oscillatory waves. Trans. Camb. Phil. Soc. 8 (1847), 441-455. 